\title{
New treatment options for fibromyalgia: critical appraisal of duloxetine
}

\author{
Nurcan Üçeyler' \\ Martin Offenbächer ${ }^{2}$ \\ Frank Petzke ${ }^{3}$ \\ Winfried Häuser ${ }^{4}$ \\ Claudia Sommer' \\ 'Department of Neurology, University \\ of Würzburg, Germany; ${ }^{2} \mathrm{GRP}$ - \\ Generation Research Program, Human \\ Science Center, University of \\ Munich, Germany; ${ }^{3}$ Department of \\ Anesthesiology, University of Cologne, \\ Germany; ${ }^{4}$ Center of Pain Therapy, \\ Klinikum Saarbrücken gGmbH, \\ Winterberg I, Saarbrücken, Germany
}

Correspondence: Claudia Sommer

Department of Neurology, University of

Würzburg, Josef-Schneider-Str. I I, 97080

Würzburg, Germany

$\mathrm{Tel}+49$ 93। 20I 23763

Fax +49 93। 20I 23489

Email sommer@uni-wuerzburg.de

\begin{abstract}
Fibromyalgia syndrome (FMS) is a chronic condition characterized by widespread pain, tender points, fatigue, and sleep disturbance. FMS leads to high disability levels, poor quality of life, and extensive use of medical care. Effective pharmacological treatment options are rare, and treatment effects are often of limited duration. Duloxetine is a new selective serotonin and norepinephrine reuptake inhibitor that is licensed for the treatment of pain in diabetic neuropathy. So far two randomized, placebo-controlled trials have investigated the short-term safety and efficacy of duloxetine $60 \mathrm{mg} /$ day and $120 \mathrm{mg}$ /day in patients suffering from FMS over a period of 12 weeks. Both dosages were superior to placebo in pain relief, and improvement in quality of life and depressive symptoms. The analgesic effect was largely independent of the antidepressant action of duloxetine. The higher dose of $120 \mathrm{mg} /$ day further reduced the tender point count and elevated the tender point pain thresholds. Only mild to moderate adverse effects were reported. Duloxetine $60 \mathrm{mg} /$ day and $120 \mathrm{mg}$ /day has proven to be beneficial in the treatment of FMS symptoms. As true for other antidepressants further studies are needed to assess the long-term efficacy and safety of duloxetine as an additional pharmacological treatment option in FMS.
\end{abstract}

Keywords: fibromyalgia syndrome, duloxetine, antidepressant, review, SNRI

\section{Introduction}

Fibromyalgia syndrome (FMS) is defined as a condition including chronic widespread pain (ie, pain in all four body quadrants for more than 3 months) and at least 11 out of 18 tender points that are painful upon digital palpation with $4 \mathrm{~kg}$. These criteria were developed by the American College of Rheumatology (ACR) (Wolfe et al 1990) to provide a consensus definition for FMS, establish new criteria for the classification of FMS, to study the relation of "primary" and "secondary" FMS, and to assess the strength of previous definition criteria. In addition to pain and tenderness, most patients with FMS suffer from accompanying symptoms like fatigue, poor sleep, gastrointestinal disturbances, anxiety, or depression. Population-based estimates of the prevalence of FMS range from $0.5 \%$ to $5.8 \%$ (Gran 2003). Women are more frequently affected than men (Wolfe et al 1995) and patients diagnosed with FMS cause considerable direct (health care use) (White et al 1999; Penrod et al 2004; Boonen et al 2005) and indirect costs (sick-leave, disability pension) (Henriksson et al 2005). Effective treatment options are therefore needed not only for medical but also for economic reasons (Robinson and Jones 2006).

Numerous pharmacologic and non-pharmacologic treatment options are offered to (Robinson and Jones 2006) and are used by patients suffering from FMS (Bennett et al 2007). To date over 500 peer-reviewed articles on the therapy of FMS have been published (Goldenberg and Smith 2003), yet no treatment has proven potent enough to alleviate the entire scope of symptoms and disabilities associated with FMS (van Koulil et al 2007) - a goal that may be unrealistic considering the variability of symptoms. 
In addition, treatment response is usually partial and limited to patient subgroups. To provide patients and physicians an orientation within the continuously growing number of studies on the pharmacological treatment of FMS, evidence-based guidelines have been published by the American Pain Society (APS) (Goldenberg et al 2004) and the European League Against Rheumatism (EULAR) (Carville et al 2008). Both gave the highest level of recommendation to treatment of FMS symptoms with antidepressants. It is important to note that none of the antidepressants investigated is licensed for the treatment of FMS. To date, the only drug licensed by the US Food and Drug administration (FDA) for the treatment of FMS symptoms is the anticonvulsant pregabalin, which was effective in two randomized, double-blind, placebocontrolled trials (RCT) (Crofford et al 2005; Arnold et al 2007). However, some antidepressants are approved for the treatment of chronic pain, and an application has been filed with the FDA for the selective serotonin and norepinephrine reuptake inhibitor (SNRI) duloxetine.

Duloxetine is a "pure" SNRI without actions on further receptors, thus avoiding cholinergic or adrenergic side effects (Bymaster et al 2001). It is a safe and efficacious antidepressant with only mild adverse effects (Detke et al 2002; Goldstein et al 2002). A recent study could show an effect of duloxetine on painful physical symptoms associated with depression (Goldstein et al 2004). Furthermore, duloxetine is effective in the treatment of painful diabetic peripheral neuropathy, independent of depression (Goldstein et al 2005; Raskin et al 2005). It is licensed for the treatment of major depression and generalized anxiety disorders in adults, and for painful diabetic peripheral neuropathy.

\section{Duloxetine $120 \mathrm{mg} /$ day reduces pain in FMS}

The effect of duloxetine on symptoms of FMS has been investigated in two RCTs. The first trial was a multi-center RCT with parallel design lasting over 12 weeks (Arnold et al 2004). Of 555 patients screened, 207 (37.3\%) were randomized. Of these, 124 (59.9\%) completed the study. The placebo group consisted of 103 patients, of whom $66(64.1 \%)$ completed the trial while the duloxetine group comprised 104 patients of whom 58 (55.7\%) completed the study. The mean age of patients was 49.9 years, $88.5 \%$ of the study population were women, and $88.5 \%$ Caucasian. Of the study population, $35.6 \%$ reported major depression.

Duloxetine was applied in a daily dosage of $120 \mathrm{mg}$ (ie, $60 \mathrm{mg}$ twice a day). Patients started with $20 \mathrm{mg}$ /day and titrated to $60 \mathrm{mg}$ twice a day within two weeks. The patients were assessed regularly at 10 visits. Paracetamol (up to $2 \mathrm{~g} /$ day) and acetyl salicylic acid (up to $325 \mathrm{mg}$ /day) were the only analgesics allowed. There was a wash out period of 7 days for antidepressants before the second visit (exceptions: monoaminoxidase inhibitors a 14-day wash out; fluoxetine a 30-day wash out). A number of exclusion criteria were observed, such as concomitant rheumatologic and cardiac diseases, the involvement in disability reviews, and failure to respond to more than two antidepressants from different classes for depression or FMS.

The main goal was to examine the efficacy and safety of duloxetine in the treatment of FMS symptoms. Primary outcome measures were assessed using the Fibromyalgia Impact Questionnaire (FIQ), which gave the FIQ total score and FIQ pain score. Secondary outcome measures were FIQ fatigue, morning tiredness, and stiffness. The Brief Pain Inventory (BPI) measured the average pain severity score over the past 24 hours and the average interference score of FMS symptoms with items such as general daily activity, mood, or walking activity. The Clinical Global Impression of Severity scale (CGIS) and the Patient Global Assessment of Improvement scale (PGAI) were further applied. Quality of life measures covered the Quality of Life in Depression Scale total score (QLDS), the Shehaan Disability Scale total score (SDS), and the Medical Outcomes Study Short Form 36 (SF-36). Furthermore, tender point number and tender point pain thresholds were assessed using an algometer.

Patients in the duloxetine group more frequently reported adverse effects (90.4\%: duloxetine group vs $74.8 \%$ : placebo group), which were mainly insomnia, xerostomia (dry mouth), and constipation. The severity of most drug-related adverse effects was mild to moderate; severe treatment-emergent adverse events were equally present in the duloxetine and placebo group. Patients treated with duloxetine had a small increase from baseline to endpoint in heart rate. No clinically relevant changes in laboratory tests were observed. There were 18 study drop outs due to side effects in the duloxetine group and 11 in the placebo group, with no significant intergroup difference.

Except for a few items (FMS associated fatigue; FIQ pain, fatigue, and tiredness on awakening scores; Beck Anxiety Inventory total score) duloxetine $120 \mathrm{mg}$ /day treatment improved most efficacy measures. Compared to placebo, patients treated with duloxetine showed significant improvement in the FIQ total and stiffness score, the BPI average pain severity and pain interference score, the CGIS, and the PGAI. Also several quality of life measures (QLDS total score, SDS total score, SF-36) improved in the duloxetine group 
compared to placebo. Furthermore, duloxetine significantly reduced the tender point count and the tender point pain thresholds. A reduction of at least $50 \%$ in the FIQ pain score was achieved in $27.7 \%$ of the duloxetine group and in $16.7 \%$ of the placebo group, but this difference failed to reach significance $(\mathrm{p}=0.06)$. Interestingly, a gender difference was found with a lack of response in men; however, this finding may be biased by the small number of male subjects in the duloxetine ( $\mathrm{n}=12$ out of 104 patients) and placebo groups ( $n=11$ out of 103 patients). It has to be noted that reduction of pain severity by duloxetine was independent of accompanying major depression, but regression analysis showed a small indirect treatment effect through improvement in depressive symptoms.

This RCT was of excellent quality reaching a Jadad score of 5 . The Jadad score is a validated numerical score ranging from 0 to 5 assigned as a rough measure of study design/ reporting quality ( 0 being weakest and 5 being strongest) (Jadad et al 1996). A power calculation was performed as well as an intention to treat analysis with further ANOVA. Data were adjusted for multiple testing and were suitable for meta-analysis.

\section{Duloxetine $60 \mathrm{mg} /$ day versus I 20 mg/day}

The second trial also was a multi-center RCT with parallel design lasting over 12 weeks (Arnold et al 2005). Of 745 patients screened, $354(47.5 \%)$ were suitable for the study and were randomized. Of these, $215(60.7 \%)$ completed the study. The placebo group consisted of 120 and the duloxetine group of 234 patients. In the placebo group, 68 participants $(56.7 \%)$ completed the trial and in the duloxetine group, 147 $(60.5 \%)$. Mean age of patients was 49.6 years, all patients were women, $89.5 \%$ were Caucasian.

Two treatment regimes were investigated. Duloxetine was applied in a dosage of $60 \mathrm{mg}$ once daily or $120 \mathrm{mg}$ (60 mg twice a day). Patients randomized for the higher dose started with $60 \mathrm{mg} /$ day and titrated to $120 \mathrm{mg}$ /day within 3 days. Patient assessment was performed during 7 visits. The permitted analgesic rescue medication consisted of paracetamol (up to $2 \mathrm{~g}$ /day), and acetyl salicylic acid (up to $325 \mathrm{mg} /$ day) for cardiac prophylaxis. All other analgesics and medication with central nervous system activity were excluded, with a wash out period identical to the first RCT. Of the study population, $26 \%$ reported current major depression. A number of exclusion criteria were observed such as concomitant rheumatologic and cardiac diseases, and being refractory to treatment, in the investigator's opinion.
The aim of this RCT was to confirm the results of the first study, and to examine further the efficacy and safety of duloxetine in the treatment of FMS symptoms including a lower dose. The primary outcome measure was pain severity as assessed by the BPI average pain severity score. Secondary outcome measures were the BPI interference score, the FIQ total score, the CGIS, the Hamilton Depression Rating Scale (HAMD), and the Patient Global Impression of Improvement (PGII). Quality of life measures covered the QLDS, the SDS, and the SF-36. Additionally, the tender point count and the mean tender point pain threshold were determined.

Patients treated with duloxetine more frequently reported adverse effects (92.4\% duloxetine $60 \mathrm{mg}$; $90.5 \%$ duloxetine $120 \mathrm{mg}$ ) than patients in the placebo group (79.2\%). Patients in both duloxetine groups reported nausea, xerostomia (dry mouth), constipation, decreased appetite, and anorexia more frequently than patients in the placebo group. Diarrhea and nasopharyngitis were reported more frequently by patients treated with duloxetine $60 \mathrm{mg} /$ day than those treated with placebo. Somnolence, increased sweating, feeling jittery, and nervousness were reported more often by patients in the duloxetine $120 \mathrm{mg} /$ day group than by those treated with placebo. More patients in the duloxetine $60 \mathrm{mg}$ /day reported insomnia during the discontinuation phase compared to placebo-treated patients. There were more study drop outs due to side effects in both duloxetine groups (about 22\%) than in the placebo group (11.7\%), while overall more patients discontinued the study in the placebo group (43\%) compared to duloxetine $60 \mathrm{mg} /$ day (35\%) and duloxetine $120 \mathrm{mg} /$ day (39\%).

In both duloxetine treatment groups significantly more patients showed a $\geq 50 \%$ decrease in the BPI average pain severity score compared to placebo (41\% duloxetine $60 \mathrm{mg} /$ day and $120 \mathrm{mg} /$ day each; 23\% placebo). Also the BPI-interference scores and the scores assessed with the FIQ, the CGIS, and the PGII decreased significantly more in the treatment groups with both dosages than in the placebo group without difference between the two dosages of duloxetine. Quality of life improved under duloxetine $60 \mathrm{mg} /$ day and $120 \mathrm{mg} /$ day. Taken together, both dosages were equally effective in reducing pain and improving patients' quality of life without significant intergroup difference, and again the reduction in pain severity was independent of accompanying major depression. For the tender points, however, different outcomes were observed with the two drug dosages. Only duloxetine $120 \mathrm{mg} /$ day significantly reduced tender point count and increased tender point pain thresholds.

This RCT was also of excellent quality with a Jadad score of 5. A power calculation was performed as well as 
an intention to treat analysis with further ANOVA. Data were adjusted for multiple testing and were suitable for meta-analysis.

\section{Summary}

FMS is a lifelong disorder, which requires long-term treatment that has to address various symptoms. The pathophysiological background is unclear and causal treatment options are not available. There is a growing body of pharmacological studies in search of the most proper drug to relieve symptoms of FMS with minimum adverse effects. The most commonly used drugs are antidepressants, which are effective in mood disorders and pain - two symptoms frequently combined in FMS. Serotonin and norepinephrine are involved in endogenous central analgesic pathways (Millan 2002) and have antidepressant action. Therefore a beneficial effect on symptoms in patients with FMS is expected using SNRI such as duloxetine. In the two RCTs on duloxetine for FMS, the investigated dosages of $60 \mathrm{mg} /$ day and $120 \mathrm{mg} /$ day were equally effective in pain relief independent of the drug's antidepressant action during the 12 weeks of observation. As secondary outcome, improvement of patients' quality of life was achieved. The higher dosage of $120 \mathrm{mg}$ /day was associated with more adverse effects, but led to an additional reduction of tender point count and tender point pain thresholds. It is important to note that the side effects in the second trial, including a high percentage of nausea, most probably were due to the different titration regimes used: while in the first trial patients started with $20 \mathrm{mg} /$ day and titrated to $120 \mathrm{mg} /$ day within 2 weeks, in the second trial patients started with $60 \mathrm{mg} /$ day and reached $120 \mathrm{mg} /$ day within 3 days. Therefore, the motto for safe duloxetine treatment should be "start low and go slow" - as is true for most antidepressants. As with every drug, contraindications and possible drug interactions should be considered before prescribing duloxetine.

In both RCTs a considerable number of inclusion and exclusion criteria were observed leading to the question of whether this might have influenced the outcome measures by generating a selected group of patients. In particular, the exclusion of patients who were obviously difficult to treat (failure to respond to more than two different classes of antidepressants for depression or FMS in the 2004 trial and being treatment refractory in the investigator's judgement in the 2005 trial) might reduce the impact of the trials for general practice.

Although the two RCTs on duloxetine give evidence for the effectiveness of duloxetine in FMS, it has to be taken into account that the reduction in the main symptom "pain" was only moderate in both studies (27.8\% and $43.8 \%)$. These percentages are in line with the moderate efficacy of all drugs tested for FMS so far, including the tricyclic antidepressant amitriptyline. However, given a side effect profile that is in part different from that of tricyclic antidepressants, SNRI may be a useful alternative in FMS. One further study investigated milnacipran, another SNRI for patients with FMS, and found efficacy in the reduction of pain when applying a daily dosage of $200 \mathrm{mg}$, althouhg no effect was seen on sleep disturbances. No severe adverse effects were reported (Vitton et al 2004). Further trials using other SNRI and trials combining drugs with different mechanisms of action should be performed, with the aim of achieving more marked pain reduction and improvement of physical function with minimum adverse effects.

As for all antidepressants investigated in the context of FMS, only short-term data are available for duloxetine, mainly collected in Caucasian women. Therefore, long-term trials and studies investigating the effect of duloxetine in male FMS patients and non-Caucasians are needed to expand the knowledge about the possible effects and side effects of this drug. Given the good tolerability and at least moderate effect on pain and quality of life in patients with FMS, duloxetine is a valuable addition to the range of pharmacological treatment options for FMS.

\section{Disclosures}

CS has received honoraria as a speaker at educational events from Lilly and Boehringer Ingelheim. The other authors have no conflicts of interest.

\section{References}

Arnold LM, Crofford LJ, Martin SA, et al. 2007. The effect of anxiety and depression on improvements in pain in a randomized, controlled trial of pregabalin for treatment of fibromyalgia. Pain Med, 8:633-8.

Arnold LM, Lu Y, Crofford LJ, et al. 2004. A double-blind, multicenter trial comparing duloxetine with placebo in the treatment of fibromyalgia patients with or without major depressive disorder. Arthritis Rheum, 50:2974-84.

Arnold LM, Rosen A, Pritchett YL, et al. 2005. A randomized, doubleblind, placebo-controlled trial of duloxetine in the treatment of women with fibromyalgia with or without major depressive disorder. Pain, 119:5-15.

Bennett RM, Jones J, Turk DC, et al. 2007. An internet survey of 2,596 people with fibromyalgia. BMC Musculoskelet Disord, 8:27.

Boonen A, van den Heuvel R, van Tubergen A, et al. 2005. Large differences in cost of illness and wellbeing between patients with fibromyalgia, chronic low back pain, or ankylosing spondylitis. Ann Rheum Dis, 64:396-402.

Bymaster FP, Dreshfield-Ahmad LJ, Threlkeld PG, et al. 2001. Comparative affinity of duloxetine and venlafaxine for serotonin and norepinephrine transporters in vitro and in vivo, human serotonin receptor subtypes, and other neuronal receptors. Neuropsychopharmacology, 25:871-80. 
Carville SF, Arendt-Nielsen S, Bliddal H, et al. 2008. EULAR evidence based recommendations for the management of fibromyalgia syndrome. Ann Rheum Dis, 67:536-41.

Crofford LJ, Rowbotham MC, Mease PJ, et al. 2005. Pregabalin for the treatment of fibromyalgia syndrome: results of a randomized, doubleblind, placebo-controlled trial. Arthritis Rheum, 52:1264-73.

Detke MJ, Lu Y, Goldstein DJ, et al. 2002. Duloxetine, $60 \mathrm{mg}$ once daily, for major depressive disorder: a randomized double-blind placebocontrolled trial. J Clin Psychiatry, 63:308-15.

Goldenberg D, Smith N. 2003. Fibromyalgia, rheumatologists, and the medical literature: a shaky alliance. J Rheumatol, 30:151-3.

Goldenberg DL, Burckhardt C, Crofford L. 2004. Management of fibromyalgia syndrome. Jama, 292:2388-95.

Goldstein DJ, Lu Y, Detke MJ, et al. 2004. Effects of duloxetine on painful physical symptoms associated with depression. Psychosomatics, 45:17-28.

Goldstein DJ, Lu Y, Detke MJ, et al. 2005. Duloxetine vs. placebo in patients with painful diabetic neuropathy. Pain, 116:109-18.

Goldstein DJ, Mallinckrodt C, Lu Y, et al. 2002. Duloxetine in the treatment of major depressive disorder: a double-blind clinical trial. J Clin Psychiatry, 63:225-31.

Gran JT. 2003. The epidemiology of chronic generalized musculoskeletal pain. Best Pract Res Clin Rheumatol, 17:547-61.

Henriksson CM, Liedberg GM, Gerdle B. 2005. Women with fibromyalgia: work and rehabilitation. Disabil Rehabil, 27:685-94.

Jadad AR, Moore RA, Carroll D, et al. 1996. Assessing the quality of reports of randomized clinical trials: is blinding necessary? Control Clin Trials, 17:1-12.
Millan MJ. 2002. Descending control of pain. Prog Neurobiol, 66:355-474.

Penrod JR, Bernatsky S, Adam V, et al. 2004. Health services costs and their determinants in women with fibromyalgia. $J$ Rheumatol, 31:1391-8.

Raskin J, Pritchett YL, Wang F, et al. 2005. A double-blind, randomized multicenter trial comparing duloxetine with placebo in the management of diabetic peripheral neuropathic pain. Pain Med, 6:346-56.

Robinson RL, Jones ML. 2006. In search of pharmacoeconomic evaluations for fibromyalgia treatments: a review. Expert Opin Pharmacother, $7: 1027-39$.

van Koulil S, Effting M, Kraaimaat FW, et al. 2007. Cognitive-behavioural therapies and exercise programmes for patients with fibromyalgia: state of the art and future directions. Ann Rheum Dis, 66:571-81.

Vitton O, Gendreau M, Gendreau J, et al. 2004. A double-blind placebocontrolled trial of milnacipran in the treatment of fibromyalgia. Hum Psychopharmacol, 19(Suppl 1):S27-35.

White KP, Speechley M, Harth M, et al. 1999. The London Fibromyalgia Epidemiology Study: direct health care costs of fibromyalgia syndrome in London, Canada. J Rheumatol, 26:885-9.

Wolfe F, Ross K, Anderson J, et al. 1995. The prevalence and characteristics of fibromyalgia in the general population. Arthritis Rheum, 38:19-28.

Wolfe F, Smythe HA, Yunus MB, et al. 1990. The American College of Rheumatology 1990 Criteria for the Classification of Fibromyalgia. Report of the Multicenter Criteria Committee. Arthritis Rheum, 33:160-72. 
